\title{
Outcomes of a service teaching module on ODEs for physics students
}

Diarmaid Hyland ${ }^{\mathrm{a}}$, b*, Paul van Kampen ${ }^{\mathrm{a}}$, and Brien C. Nolan ${ }^{\mathrm{b}}$

${ }^{a}$ Centre for the Advancement of STEM Teaching and Learning and the School of Physical Sciences, Dublin City University, Dublin, Ireland; ${ }^{b}$ Centre for the Advancement of STEM Teaching and Learning and the School of Mathematical Sciences, Dublin City University, Dublin, Ireland.

Email address of corresponding author: Diarmaid.hyland3@mail.dcu.ie

Email address of other authors: $\underline{\text { Paul.van.kampen@dcu.ie }}$ Brien.nolan@dcu.ie

ORCID Identifiers:

Paul van Kampen: orcid.org/0000-0003-3043-8110

Brien Nolan: orcid.org/0000-0002-9356-6833

Diarmaid Hyland: orcid.org/0000-0003-3787-5153 


\section{Outcomes of a service teaching module on ODEs for physics students}

\section{Abstract}

This paper reports on the first part of a multiphase research project that seeks to identify and address the difficulties encountered by physics students when studying differential equations. Differential equations are used extensively by undergraduate physics students, particularly in the advanced modules of their degree. It is, therefore, necessary that students develop conceptual understanding of differential equations in addition to procedural skills. We have investigated the difficulties encountered by third-year students at Dublin City University in an introductory differential equations module. We developed a survey to identify these difficulties and administered it to students who had recently completed the module. We found that students' mathematical ability in relation to procedural competence is an issue in their study of differential equations, but not as severe an issue as their conceptual understanding. Mathematical competence alone is insufficient if we expect our students to be able to recognize the need for differential equations in a physical context and to be able to set up, solve, and interpret the solutions of such equations. We discuss the implications of these results for the next stages of the research project.

Keywords: conceptual understanding; instrumental understanding; service teaching; differential equations. 


\section{Introduction and Background}

In this paper, we report on the first part of a multiphase study in which we study how a service taught mathematics module prepares physics students for using ordinary differential equations (ODEs) in their discipline. Here we explore students' instrumental and conceptual understanding of ODEs after they have completed a standard module on this topic. The module carries 5 ECTS credits and is taught by mathematicians at Dublin City University (DCU), Ireland. Service teaching may be defined as teaching disciplinary expertise resident in one school to students enrolled in a degree programme coordinated by a different school [1]. Service teaching of mathematics sometimes (perhaps often) involves students solving exclusively decontextualized problems with little to no emphasis on how the mathematics they are learning can help them with the study of their own discipline [2]. Not many students show an ability to use the mathematics they have learned in their chosen discipline, even if they performed well in the mathematics module [2-5].

Nankervis [6] carried out a review of service teaching at Royal Melbourne Institute of Technology (RMIT) that has several interesting findings. It stressed that the issues that arise are not with service teaching itself (which has many potential benefits) but with several surrounding factors that influence students' levels of satisfaction with service taught modules. The study highlights the quality of teaching and learning, assessments, and the relevance of the content of service taught courses to the students' own discipline as reasons for dissatisfaction among students.

In the case of service teaching mathematics to physics students, a study by Caballero et al. [7] found that students frequently struggle to connect mathematics with physics in subsequent physics modules. Thompson, Bucy, and Mountcastle [8] identified the phenomenon of students successfully applying and manipulating mathematical equations but being unable to generate or interpret these equations. As observed by Stephan and Rasmussen [9], when a course concentrates on algorithmic methods to solve differential equations, it leaves less time to highlight applications and understanding of the mathematics; this makes the transition to using the mathematics in physics more difficult. Redish and Kuo [10] observed that mathematicians and physicists make meaning with mathematics in different ways. For this reason, using the language of mathematics in physics is not straightforward. Manogue and Dray [11] also used a language metaphor and posited that mathematics and physics courses use a different dialect of the same language. It has been observed that the aims of mathematicians 
and physicists can be very different: service taught mathematics modules on ODEs tend to emphasise the classification of differential equations and their theoretical aspects (questions of existence and uniqueness of solutions). Techniques for solving differential equations are also studied.

By contrast, in physics modules, modelling often is emphasized $[5,10,11]$, because there are numerous models in physics where the value of a quantity and changes in that quantity are related. For example, the speed of an object depends on its acceleration; the radioactivity of a sample depends on the amount of the sample present; the change in temperature of a body depends on the difference in temperature between the body and its environment. Except in highly idealised settings, the analysis of these and numerous other cases requires students to recognise the need for the use of a differential equation, to set up the appropriate differential equation, to solve it, and to interpret the solution. In addition, the study of ODEs underpins the study of partial differential equations (PDEs) which also have a significant role in undergraduate physics (heat equation, wave equation, and Maxwell's equations for example). Thus, students must not only apply mathematical knowledge to solve a differential equation, but also should recognise the mathematical structure of the problem at hand being suitably described by a differential equation. There is a clear difference in the use of differential equations in mathematics and physics modules, and differences in purpose of mathematics and physics teachers may well compound problems the students may have with solving ODEs per se.

To assess the degree of the problem at DCU, a survey of academic staff in the School of Physical Sciences was conducted. The survey asked lecturers about the use of differential equations in their modules and how student ability plays a role in their modular design. The responses from this survey indicated that the role of differential equations in advanced physics modules has diminished to the point of omission in certain cases. Lecturers feel hamstrung by the students' inability to apply the differential equations they are learning in mathematics modules to the physics they study as part of their degree program. The issues cited in the survey represent areas for potential improvement of service teaching in DCU and further afield.

Much of the research on the teaching and learning of differential equations focuses on the following themes: the effect of a change in pedagogy on conceptual understanding [12-15], students' conception of the solution to a differential equation [16, 17], and introducing a 
geometric approach to solving differential equations [18, 19]. Of the work on change in pedagogic approach, Rasmussen and Kwon [20] reported on the Inquiry Orientated Differential Equations (IO-DE) project, in which they adapted the instructional design theory of Realistic Mathematics Education or RME [21] for use at the undergraduate level. At the heart of RME is an emphasis on real world problems in the mathematics classroom. Their results compared favourably to the traditional methodologies used previously, as measured via pre- and posttesting.

All of this feeds into the desire to improve tertiary physics education and mathematics education, the experience of students taking these courses, and their knowledge and understanding of their subjects.

The research described in this paper is the initial stage of a multiphase research project that aims to identify and address the difficulties encountered by physics students in their study of differential equations. We have focused on a group of students who have completed a typical 12-week introductory service module on differential equations (MS225). The physics students at DCU complete MS225 in the third year of their study and begin to see differential equations appear in advanced physics modules from this time onward. Having found a suitable environment for our study, we have focused on the following research questions:

1) Do our students have the necessary instrumental understanding ${ }^{1}$ in the following areas to succeed in their study of ODEs?

a) Manipulation of exponentials in equations

b) Evaluating indefinite integrals

2) Do our students have a well-developed concept image ${ }^{1}$ of ODEs upon completion of this module?

a) When presented with an ODE, what is brought to mind?

b) What do students know about ODEs and their applications after completing their module?

c) Do they understand what a solution to an ODE is?

We stress again that at this stage of the multiphase project we have not yet changed our

1. The terms 'instrumental understanding' [22] and 'concept image' [23] will be discussed in the Methodology section. 
teaching approach; rather, the results from this study on a module taught in a standard way have informed the development of future interventions. In this paper we report on a study of the students' instrumental and relational understanding of ODEs after taking a standard service taught module. The research questions reflect that we value both types of understanding. Instrumental understanding relates to students' ability to complete the mathematical tasks they encounter during this module. This makes it a key component of this study. We have investigated our students' conceptual understanding through establishing their concept image of ODEs. When investigated in conjunction with the students' concept image instrumental understanding will provide a more detailed account of their areas of strength and weakness in relation to their understanding of differential equations. Our decision to focus on manipulation of exponentials in equations and evaluating indefinite integrals is based on the experience of the research team delivering and tutoring modules on ODEs in the past.

\section{Methodology}

The research described in this paper is the initial stage of a multiphase research project. While the entire project adopts a mixed methods research design [24], this paper focuses on the first stage of data collection which comes from a Diagnostic Survey. The Diagnostic Survey (described below) is designed to gather data related to the research questions stated above.

Before describing the data collection and analysis, it is necessary to explain what we mean when we refer to concept image and instrumental understanding in our research questions. When we refer to concept image in our second research question, we are referring to Tall and Vinner [23] who defined the concept image as 'the total cognitive structure that is associated with the concept, which includes all the mental pictures and associated properties and processes'. In order to assess our students' concept image, we designed open-ended questions that encouraged them to explain terms to us as they understand them. Concept image has previously been used to investigate students' understanding of mathematics in physics [2527] and we believe that it provides an appropriate lens to study students' understanding of ODEs. The data generated from the corresponding questions in the Diagnostic Survey will give us the best opportunity to identify certain aspects of students' strengths and weaknesses in relation to ODEs. We agree with Tall and Vinner [23] that what students write down or otherwise communicate to us is not necessarily their entire concept image, since the question may not have cued them to share everything with us. What we obtain from students' answers is called their 'evoked concept image' [23, p. 152]. 
In our first research question, we refer to terminology first used by Skemp [22]. In this paper, Skemp talks about instrumental understanding as having 'rules without reasons'. In describing and assessing students' instrumental understanding in relation to general calculus and algebra skills, we are assessing their ability to systematically solve problems independent of context, and independent of any necessity to explain or validate their reasoning: this is instrumental understanding. In the same paper, Skemp talks about relational understanding as 'knowing both what to do and why'. Our research questions are best described and answered using concept image and instrumental understanding, but the dichotomy presented by Skemp is noteworthy. We also note a connection with Sfard's identification of a duality (as opposed to a dichotomy) in relation to mathematical concepts as having complementary aspects referred to as operational and structural [28]. We will return to this duality below in our discussion of the results of this research.

The nature of the research questions necessitates the collection of both qualitative and quantitative data for analysis. The quantitative data will be students' answers to questions assessing instrumental understanding. The qualitative data was analysed using the general inductive approach described by Thomas [29].

\section{Administration and data collection}

The Diagnostic Survey was administered to students during a lecture slot in the final week of the semester and comprised two sections. Students were given twenty minutes to complete each section and were given one section at a time. No student needed extra time to complete their work. They were also informed that if they did not know the answer to a question that they should state this on their sheet and attempt the next question, to allow us to differentiate between students who were unable to complete the questions and students who had insufficient time to do so. The students worked alone and were not allowed to use notes or other resources during the Diagnostic Survey.

\section{Instrument}

We designed a Diagnostic Survey to answer the research questions outlined in the introduction. The first section of the Diagnostic Survey focused on the first set of research questions and the second section of the Diagnostic Survey focused on the second set of research questions. In the survey and throughout the module the term 'differential equation' was used as synonymous with ODE. 
Section 1

Q1. Find $x$ and $y$ if

- $5=3+x$

- $4=3+x e^{-y}$

Q2. Simplify the following.

$\frac{(-\mathrm{k})^{2}}{\mathrm{k}^{-1}}+\left(\mathrm{k}^{2}+\mathrm{k}^{1}\right)^{2}$

Q3. Integrate each of the following;

- $\int\left(x^{2}+\frac{1}{x^{3}}\right) \mathrm{d} x$

- $\int \frac{1}{x} \mathrm{~d} x$

- $\int x e^{x} \mathrm{~d} x$
Section 2

Q1. Write down everything you can think of when you see each of the

following. (note: $\mathrm{C}$ is a positive constant in each case)

- $\frac{\mathrm{d} N}{\mathrm{~d} t}=-\mathrm{C}$

- $\frac{\mathrm{d} N}{\mathrm{~d} t}=-\mathrm{CN}$

- $\frac{\mathrm{d} N}{\mathrm{~d} t}=-\mathrm{C} t$

Q2. What are differential equations, and why are they useful?

Q3. Write down everything you know about the solutions to differential equations.

Figure 1: The Diagnostic Survey

Section 1 was divided into three questions; Question 3 comprised three parts (see Figure 1). The questions that appeared in this section are a selection of closed mathematical tasks that arise during the course of the module. They are not claimed to be an exhaustive list of problem areas for students but were selected for inclusion by the research team based on their relevance to the module, the related literature, and prior teaching experience.

Question 1 presents the students with a system of two equations they are asked to solve for $x$ and $y$. We chose this question because an isomorphic system of equations occurs when students solve a problem based on Newton's Law of Cooling. Newton's Law of Cooling models how the temperature of an object changes with time using a first order ODE. Problems of this form appear in MS225 and in many modules on ODEs. Successful completion of this question requires an instrumental understanding of both manipulating exponential functions and isolating an unknown when it is an exponent.

Question 2 assesses students' instrumental understanding of exponents. Classroom observations had signalled to us that manipulating exponents (specifically negative ones) cause some students difficulties. Manipulating exponents can also affect students' ability to apply the power rule of integration successfully and including a question that assesses students' instrumental understanding of exponents will shed light on both of these potential difficulties 
and potentially allow us to distinguish between both.

Question 3 was a three-part integration question involving the calculation of indefinite integrals. Integration is a key procedure when solving ODEs and students will need to be able to calculate a range of anti-derivatives throughout this course and in their future studies.

The first part of Question 3 asked students to integrate two terms for which the power rule of integration works. The second part of this question required students to evaluate $\int \frac{1}{x} d x$, a very common calculation that students will meet repeatedly when studying ODEs. The final part asked students to integrate $x e^{x}$. Students have been exposed to integration by parts and one would expect students to apply this approach in this canonical integration-by-parts exercise, but other methods, such as the method of undetermined coefficients, are possible. Integration by parts often appears when solving first order linear ODEs. It is clearly more difficult operationally than the other two integrals and could conceivably cause students difficulties, which warrants its inclusion.

Section 2 of the Diagnostic Survey contained five questions in total, three of which are described and reported on in this paper. This section was designed to identify how students view and understand ODEs upon completion of MS225 by evoking their concept image. The questions are open-ended to allow students answer as they saw appropriate.

Question 1 of Section 2 of the Diagnostic Survey asked students to write down everything they can think of when they see each of the following equations:

$$
\frac{\mathrm{d} N}{\mathrm{~d} t}=-\mathrm{C} \quad \frac{\mathrm{d} N}{\mathrm{~d} t}=-\mathrm{C} N \quad \frac{\mathrm{d} N}{\mathrm{~d} t}=-\mathrm{C} t
$$

where $\mathrm{C}$ is a positive constant in each case. Question 2 asked students what differential equations are, and why are they useful, and Question 3 asked students to write down everything they know about solutions to differential equations. These questions were designed to evaluate the students' evoked concept image in the same manner the evoked concept images of function [24], integration [25], and divergence, gradient, and curl [26] have been elicited previously.

\section{Results and Discussion}

In this section, we present the results of the Diagnostic Survey. We will begin by reporting the students' answers to the mathematical questions that made up Section 1 before describing the 
answers given to Section 2. Eighteen students completed both sections of the Diagnostic Survey.

\section{Section 1: Instrumental understanding}

Research Question 1a: manipulating exponentials in equations

As mentioned in the previous section of this paper, the questions in Section 1 evaluate instrumental understanding. Our analysis focused on how many students got each question right and what errors occurred in the incorrect answers. Question 1 required students to solve a system of non-linear algebraic equations for $x$ and $y$. Ten students (56\%) obtained the correct values for $x$ and $y$. The eight (44\%) students who were unsuccessful all obtained a correct value for $x$ and reduced the problem to solving $\frac{1}{2}=e^{-y}$, but failed to correctly isolate $y$.

We conclude that just over half of our students are able to manipulate exponentials sufficiently well for the purposes of our differential equations module, but a significant minority have not yet mastered the use of the natural log function vis a vis its relationship with exponentials.

\section{Research Question 1b: evaluating indefinite integrals}

Question 3 comprised a three-part integration question that was answered with varying levels of success. The second part of this question was answered best by students, with seventeen of the eighteen students $(94 \%)$ integrating $\frac{1}{x}$ correctly. Nine students $(50 \%)$ integrated $x^{2}+\frac{1}{x^{3}}$ correctly. One student did not give an answer and the remaining eight students failed to integrate the $\frac{1}{x^{3}}$ term correctly. One student had the correct term with an incorrect sign, another student left the $\frac{1}{x^{3}}$ term unaltered, three students arrived at $\ln x^{3}$ as their answer, and the final three students made errors when manipulating exponents during the integration process.

We attribute the difficulties encountered when integrating the $\frac{1}{x^{3}}$ term to a large extent to difficulties with negative exponents. The answers to Question 2 corroborate this view. Only seven students (39\%) answered the question correctly; of the eleven students who were incorrect, ten students made an error manipulating the expression $\frac{(-k)^{2}}{k^{-1}}$. The responses to both Questions 2 and 3 suggest that our students find manipulating negative exponents more difficult than positive ones. Table 1 shows how the students' answers to Question 2 and 3 are linked. 
All 7 students who answered the exponents question correctly also answered the integration question correctly; of the 9 students who answered the exponents question incorrectly, 7 answered the integration question incorrectly, and 2 were correct. The students who answered 'do not know' and left the question unanswered also integrated incorrectly.

Table 1: Correlation between answers to Q2 and Q3.

\begin{tabular}{|l|c|c|}
\hline exponents -- integration & correct & incorrect \\
\hline Correct & 7 & - \\
\hline Incorrect & 2 & 7 \\
\hline don't know & - & 1 \\
\hline Blank & - & 1 \\
\hline
\end{tabular}

The final part of Question 3, the integration of $x e^{x}$, was answered correctly by nine students $(50 \%)$. Of the nine students who were incorrect, two attempted to integrate by parts but did not complete their calculation; four students used the product rule for differentiation, suggesting a potential confusion between differentiation techniques and integration techniques; and three students combined various incorrect integration and differentiation algorithms.

We conclude that our students' ability to evaluate indefinite integrals is probably not at the level required. These results were obtained on completion of the module, and about half of the students were not able to integrate correctly two integrals they would have encountered frequently in the module, and beforehand in other modules. Unsatisfactory as this is, we note that this need not impede students' progress with acquiring broader or deeper knowledge of ODEs, since these difficulties occur towards the end of the process of solving ODEs (and may even go undetected).

\section{Section 2: Concept Image}

Questions 1, 2, and 3 of Section 2 enabled us to assess students' concept image of ODEs upon completion of the module and answer Research Questions 2a, b, and c respectively. The openended nature of the questions implied that the answers given by students could take several 
different forms. Using a data analysis technique described by Thomas [29], we were able to reduce the data to the form given in Tables 2 and 3, which contains information about the form, and content of answers given for each question in this section. Students typically gave isolated examples of the uses of differential equations which lent itself to a tallied display.

\section{Research Question 2a: Concept image evoked by particular ODEs}

In answering Question 1 the most common approach of students was to attempt to solve the three ODEs analytically. For the first ODE, ten students (56\%) did just that. In every case, students attempted to solve the equation by separating variables, which they succeeded in; eight of these completed the solution but provided no commentary, explanation, or validation for their approach. Another analytical approach, taken by two students (11\%), was to differentiate with respect to $t$ (Figure $2 \mathrm{a}$ and $2 \mathrm{~b}$ ). They may have been prompted to differentiate when they saw a derivative. The other answers given by students included four students (22\%) explaining that $N$ decreases at a rate $C$ with respect to time. Two students (11\%) graphed $N$ vs $t$ to show that the slope was constant. 


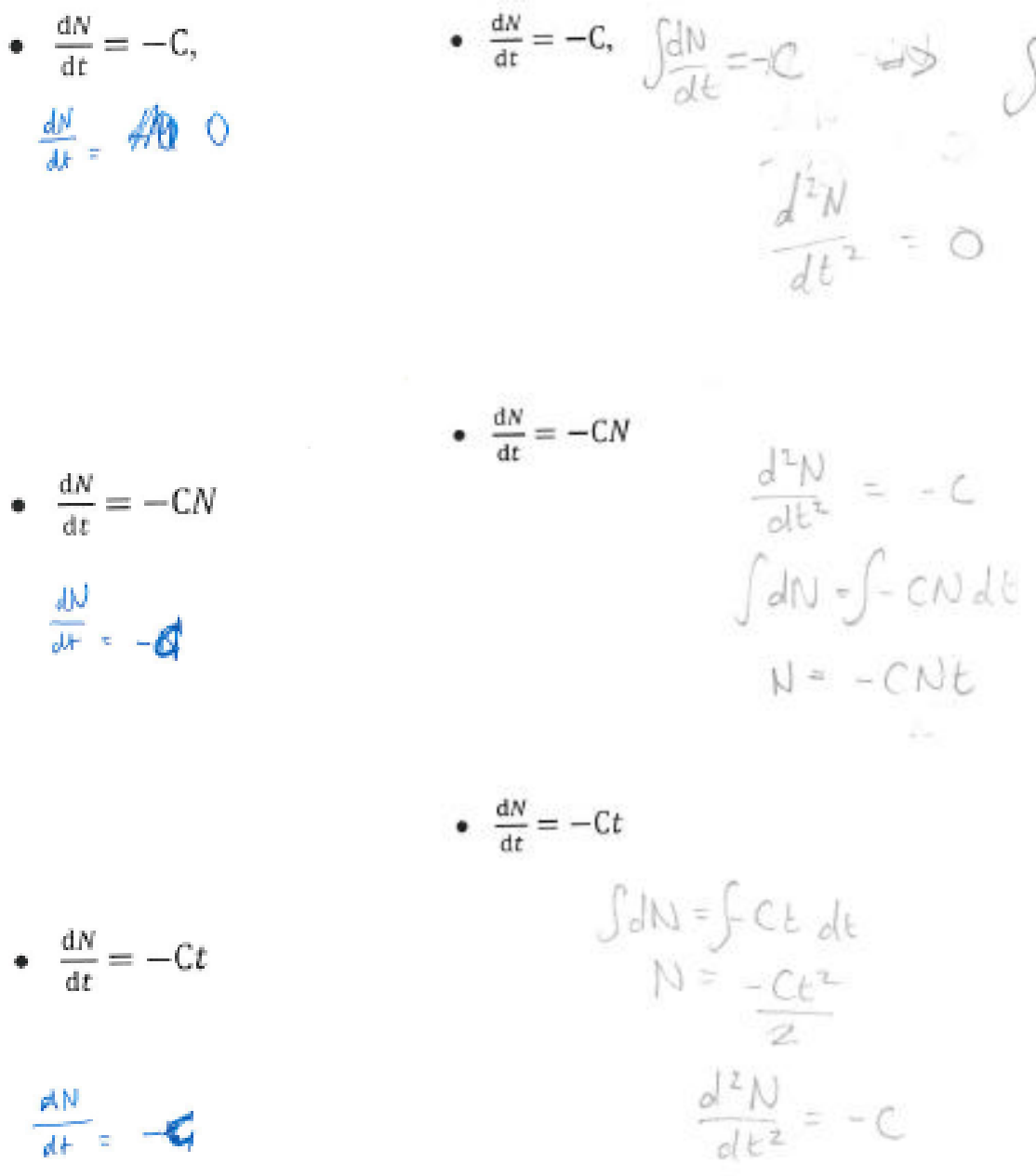

Figure 2a and 2b: Student responses to Question 1

The other ODEs of Question 1 were answered in a similar manner by the students, with answers occurring with similar frequency. Of note is that the number of students who attempted to separate and solve the given equation increased from the first equation $(n=10 ; 56 \%)$ to the third equation $(n=11 ;(61 \%))$ to the second equation $(n=13 ;(72 \%))$. While the sample is too small to make definitive statements, this finding suggests that students may identify an equation as a differential equation more easily when the dependent or independent variable is present. In this case, students identified the equation that contained the dependent variable (the second) as a differential equation most frequently. Fewer students identified the equation that contained the independent variable (the third equation) as a differential equation, and fewer still identified the equation that contained neither variable (the first equation) as a differential equation. This suggests that structure may play a part in a students' ability to recognise ODEs. Also of note was the frequency with which these ODEs caused students to mention differentiation. Figure $2 \mathrm{a}$ shows a response in which only the righthand-side of the equation was differentiated; Figure 
2(b) shows correct differentiation of both sides of the equation.Mentioning differentiation or derivatives was most frequent in the first ODE, where talk of differential equations was least frequent. It is understandable that these terms do not always appear together. In order for a student to respond in both ways, it would require them to think of a derivative as representing a process that has taken place (a function has been differentiated) at the same time as thinking of an equation where the derivative is simply another term. This can be understood in terms of Sfard's notion of duality.

\section{Research Question 2b: ODEs and their uses}

Question 2 in this section yielded varying levels of responses from students regarding their understanding of differential equations and their uses. While eight students (44\%) described differential equations as pertaining to rate of change, only one student $(6 \%)$ offered a formal definition in response to the question. Perhaps more significant is the number of students $(n=6$; $33 \%$ ) who left their survey blank or wrote 'I don't know' in response to what a differential equation is - at the end of a twelve-week module on ODEs. This strongly suggests that these students had a purely instrumental view of ODEs.

Students offered uses for differential equations more readily, with the frequency some terms were mentioned indicated in Table 2.

Table 2: Students' concept images of what ODEs are for.

\begin{tabular}{|l|c|}
\hline Use & Frequency \\
\hline Population & 5 \\
\hline Predict & 5 \\
\hline Used to mathematically model complex systems & 2 \\
\hline Stock market & 1 \\
\hline Weather (chaos) & 1 \\
\hline Optics & 1 \\
\hline Semiconductors & 1 \\
\hline Physical systems & 1 \\
\hline Acceleration & 1 \\
\hline Chemical reactions & 3 \\
\hline
\end{tabular}


Five of the students (28\%) did not explain why differential equations may be useful or where they may be applied; two of these did not give a response to the question what a differential equation is. Even among the thirteen (72\%) students who we considered to have answered the question, only two students (11\%) offered satisfactory definitions (Figure 3). The answers shown in Figure 3 were both considered to be correct but represented two different ways of understanding differential equations. Answer (a) is more succinct and uses more formal language to explain what differential equations are. This is close to the definition given to students in lectures (an ODE is an equation involving one or more derivative of a function, say $y$, which depends on a single variable, say $x$ ). Answer (b) is more descriptive and describes differential equations with reference to many examples. Both are valid answers but the second answer could be considered more desirable for a physics student.

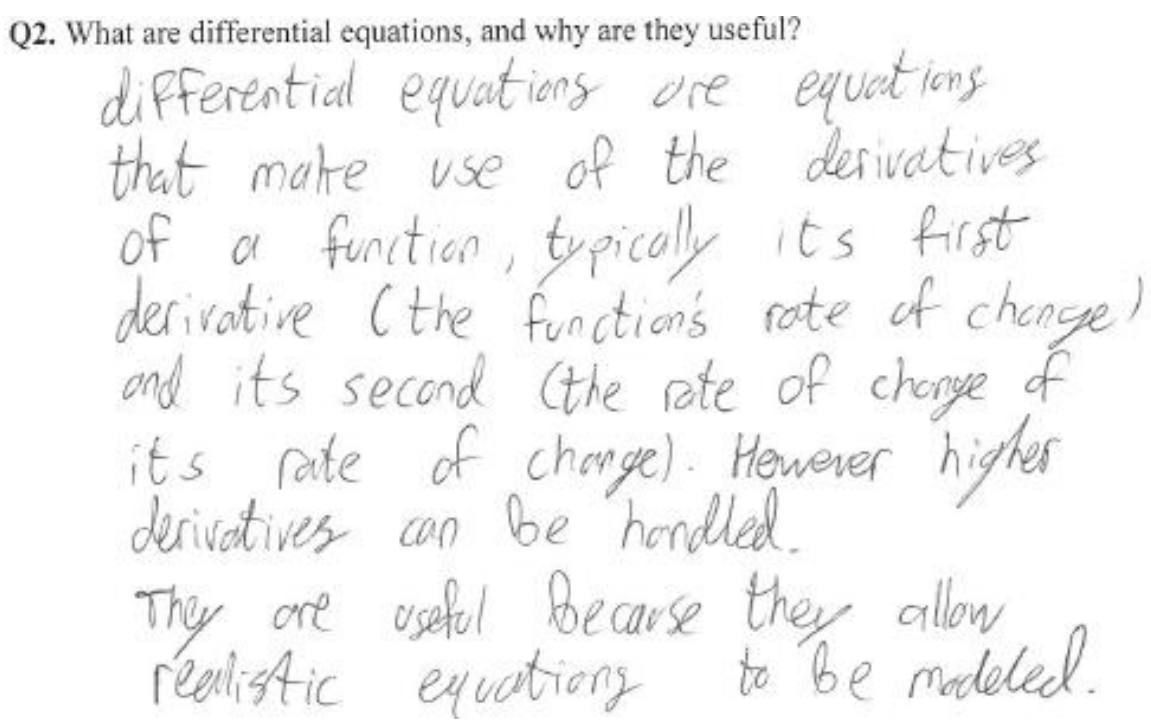

Figure 3a: Student response to Question 2 
Q2. What are differential equations, and why are they useful?

Diffepential equations are equations reppesenting the rate of change of a poraneter of a system on the study of how a system evolves. They can be used to model chemical reactions, plysical systems ( perdulum $\rightarrow$ damped, driver), the weatter, population (bacteria for example) and many theas. They can be used to mathematically model corplex systems with varying degrae of arcount (like air resistance for a pendulian). This is useful to predict the evolution of a system (if it can be approximated) if even for a short tine for chaotir systeris (eg weather).

Figure 3b: Student response to Question 2

\section{Research Question 2c: Solutions to ODEs}

Question 3 asked students to write down everything they know about solutions to differential equations. The breakdown of terms and the frequency with which they were mentioned is shown in Table 3.

Table 3: Students' concept images of solutions of ODEs.

\begin{tabular}{|l|c|}
\hline Answer & Frequency \\
\hline General solution & 7 \\
\hline Initial condition & 7 \\
\hline Contain a constant & 3 \\
\hline Differentiation & 3 \\
\hline Integration & 3 \\
\hline Numerically/analytically & 3 \\
\hline Particular solution & 3 \\
\hline Homogenous/non-homogeneous & 2 \\
\hline Not all differential equations have solutions & 2 \\
\hline Different methods e.g. Euler/Runge-Kutta & 1 \\
\hline Rate of change of something & 1 \\
\hline Tangent to a curve & 3 \\
\hline
\end{tabular}


The students' propensity toward procedures and solution techniques is clearly evident from Table 3 where terms associated with answering questions dominate the top of the table and can be seen throughout (e.g. mentioning numerical and analytical as two ways of obtaining solutions and mentioning various methods for solving DEs). Figure 4 shows a typical student response (the fact that particular solutions are not always asked for in questions explains its position further down the table). It is also striking that some students give isolated terms in a disjointed manner. An example is the discrepancy in frequency between related terms: for example, to an expert the term 'initial condition', mentioned seven times, would go hand in hand with 'particular solution', but particular solution as an answer is only seen three times; and none of the students linked the two explicitly.

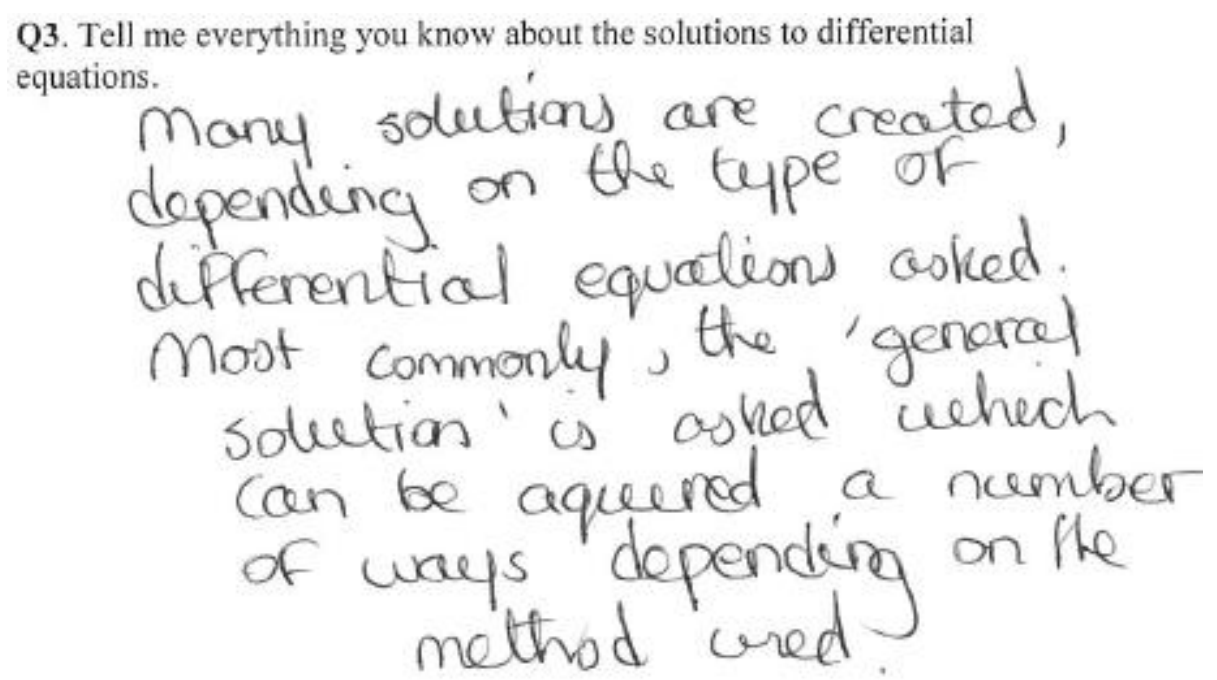

Figure 4: Typical student response referring to terms associated with solving problems

A fundamental aspect of the solution of an ODE was mentioned by only one student (6\%): that they are functions. Difficulty grasping that a function can be a solution to an equation is noted by Mallet and McCue [12] and is in keeping with the findings of Rasmussen [17 p. 66] who posited that 'this switch from conceptualizing solutions as numbers to conceptualizing them as functions is non-trivial for students'. This contrasts with the frequency with which 'contain a constant' is mentioned - again, to an expert these ideas are linked. This further suggests a focus on algorithms and procedures: awareness of the fact that solving an ODE (where including a constant in an indefinite integral may be required) produces a function is not central to the relevant procedure.

One useful way to read the results of Section 2 of the survey is in terms of Sfard's 
identification of the dual process-object nature of mathematical concepts [28]. Almost all of the students' responses refer to processes related to differential equations and their solutions. Only one student noted that the solution of an ODE is a function - thus alluding to the concept of 'solution of an ODE' as a mathematical object. It seems that for many of our students, the presence of the derivative symbol evokes the process of differentiation, or even further, is taken as a prompt to differentiate something. They struggle to understand the symbol $\frac{d y}{d x}$ as an object that can enter into a mathematical equation the way it does in an ODE. The poor conception on the part of the students of differential equations and their solutions as mathematical objects (or structures) is in line with Sfard's observation that 'in the process of concept formation, operational conceptions would precede the structural' [28 p. 10]. Attaining this type of understanding, through the processes of interiorization, condensation and reification is inherently difficult. But, as Sfard further notes, a structural understanding and approach is beneficial, allowing as it does 'more room... for more information' [28 p. 28]. From this point of view, it is perhaps not surprising that our students struggle to apply differential equations in physics: such an application is predicated on understanding ODEs as tools (objects) that can be applied in different situations.

\section{Conclusions}

The purpose of the Diagnostic Survey was to give us an overall picture of students' concept image and instrumental understanding of ODEs on completion of a standard service taught module. Our first research question looked at students' instrumental understanding of indefinite integrals and manipulation of exponentials. Having analysed the results to Section 1 of the Diagnostic Survey, we concluded that there are areas where students' instrumental understanding is not strong enough to successfully complete the level of questions on differential equations they encounter during this module. Although the majority of students were able to complete Question 1, a significant minority of students (44\%) showed an inability to use the natural $\log$ function as the inverse of the exponential function which was necessary to complete the task. A similar level of success was observed in Question 3 where only half of the students successfully integrated $\frac{1}{x^{3}}$. We believe the high level of difficulty experienced by students is because of the requirement to manipulate negative exponents. This is corroborated by the results of Question 2 of Section 1 of the Diagnostic Survey which also required students to manipulate negative exponents as part of the correct solution. All of the students who could 
manipulate negative exponents in Question 2 were able to integrate $\frac{1}{x^{3}}$. Question 2 was the only question where less than half of the students were correct, but the results of Section 1 as a whole suggest students' instrumental understanding needs significant improvement if students are going to excel in their use of differential equations.

The second research question investigated students' concept images of ODEs upon completion of MS225. The results show that students possess fragmented concept images and their understanding is at the level of processes rather than concepts. This aligns with Sfard's notion of the process-object duality of mathematical concepts [28]. Many studies report on first-year students' poor conceptual understanding in introductory calculus modules [30] but the problem is also evident for third-year students in this module on ODEs. If the conceptual understanding of the students completing this module is not greatly improved upon, using differential equations to model situations in physics effectively will likely continue to be a challenge for them. We will now consider the implication of these findings for teaching ODEs.

\section{Implications for Teaching}

The issues discussed in the results section need to be addressed in order to maximise students' ability to correctly apply ODEs in their future studies. While students who completed a standard service taught module demonstrated instrumental understanding in their assessment, there was still a lack of understanding of ODEs that prevents their physics lecturers from including material on future courses as described in the introduction. This lack of understanding, illustrated by poorly developed concept images, was evident in the results from the Diagnostic Survey. The identification of shortcomings in student understanding begins the next stage in this research project: designing an intervention that seeks to address the difficulties encountered by physics students in studying ODEs.

The intervention, which we will report on in a subsequent paper, has taken the form of a set of tutorials delivered to students in conjunction with the lectures for MS225. They replaced the tutorials that were given previously, but did not affect the module in any other way. Their content was decided upon based on results from the Diagnostic Survey and findings from relevant studies. This set of tutorials focused on in the following findings from the Diagnostic Survey:

1. Section 1 of the Diagnostic Survey highlighted some issues relating to exponents, 
algebraic systems, and integration. While not all students had difficulties with each of these concepts, an instrumental understanding of each is required to solve the ODEs encountered by these students. The intervention addressed these areas when dealing with students' mathematical ability.

2. The results of the Diagnostic Survey pointed towards students' conceptual understanding of ODEs as an area in need of improvement. Specifically, ODEs, their solutions, and the function nature of their solutions were areas in relation to which we felt that students may be able to develop a more comprehensive concept image. We have therefore devoted time in the intervention to further developing students' concept image. Redish and Kuo [10] talked about the need for physics instructors to foster components of conceptual understanding (mathematical fluency in a physical context, mathematical modelling of physical systems) in their students. While this is certainly true, there is no reason why mathematics instructors can't work towards the same goal which will allow students to succeed in using mathematics in physics.

3. Prior to the roll-out of the intervention introduced as part of this research project, the tutorials delivered as part of MS225 took a traditional format. A problem sheet was made available to students and they attended a fifty-minute recitation session each week. The research team believe that a change in pedagogical approach might foster better learning for our students. Of the research on the teaching and learning of differential equations, Rasmussen and Kwon [20] deals most directly with the manner in which content is delivered. Their paper concentrates on improving the method of teaching, resulting in The Inquiry Orientated Differential Equations (IO-DE) project which was described in the introduction as adapting the instructional design of RME. RME also placed an emphasis on embedding real-world problems in teaching. A similar attempt to emphasise real world problems in the mathematics classroom is being undertaken at the University of Leeds [31] who have changed the structure of their MATH 1400 module to include more mathematical modelling and problem-solving. This is particularly beneficial to students studying physics who would benefit from the relevance of the problem settings. Both of these projects are prime examples of successfully changing an instructional design at tertiary level. This project aims to do the same. The tutorials will use guided inquiry worksheets and adopt a cooperative learning pedagogical approach. This is informed by previous studies that reported 
success with similar goals to ours [26, 32]. This approach is also supported by McDermott and Shaffer [33], who compared the effectiveness of guided inquiry tutorials to traditional instruction using a control study.

4. The inclusion of graphical representations was considered when designing the tutorials. The point is made by Stephan and Rasmussen [9] that graphing plays a larger role in the teaching and learning of DEs than is typically seen in other maths modules. They mention graphical representations as a third method along with numerical and analytical techniques for the study and application of DEs. The authors talk about the importance of teaching this in a 'bottom-up' manner which is in keeping with the RME approach that influenced the IO-DE project. This led to the inclusion of direction fields in the intervention to help develop the third method for students, who until now have focused on analytical techniques to solve problems.

In this paper, we have described the initial stage of a multiphase research project that aims to identify and address the difficulties encountered by physics students in their study of differential equations. We designed and administered a Diagnostic Survey to students who completed a service mathematics module on differential equations. The results of the Diagnostics Survey identified areas students excel in and struggle with in their studies. This will inform the next stage of the research: the design of an intervention that seeks to address the areas of difficulty for students. Some details of the content and pedagogy used in the intervention are also provided. A comprehensive description of the design and implementation of the intervention will be explained in detail in a future paper.

\section{Acknowledgements}

We thank Mieke De Cock of KU Leuven for a thorough constructive discussion of an early draft of this paper.

\section{Funding}

This work was supported by the National Forum for the Enhancement of Teaching and Learning in Ireland [GOIPG/2014/1529]. 


\section{Reference List}

[1] Crotty M, Eklund E. History as service teaching possibilities and pitfalls. History Australia 2006;3(2):1-47.

[2] Britton S, New PB, Sharma MD, et al. A case study of the transfer of mathematics skills by university students. Int J Math Educ Sci Technol. 2003;36(1):1-13.

[3] Cui L, Rebello NS, Fletcher P, et al. Transfer of learning from college calculus to physics courses. 2006. In: Proceedings of the NARST 2006 National Meeting. San Francisco, California. [Online]. Available from:

https://www.researchgate.net/profile/N_Sanjay_Rebello/publication/242297311_T RANSFER_OF_LEARNING_FROM_COLLEGE_CALCULUS_TO_PHYSICS COURSES/links/0c960532638f5bfd86000000.pdf [Accessed 1 June 2017]

[4] Tuminaro J. A cognitive framework for analyzing and describing introductory students' use and understanding of mathematics in physics. Ph.D. thesis, Maryland. 2004.

[5] Yeatts FR, Hundhausen JR. Calculus and physics: Challenges at the interface. Am J Phys. 1992;60;716-721.

[6] Nankervis K. Service Teaching: Student experiences, issues and future directions at RMIT. 2008.

[7] Caballero MD, Wilcox BR, Doughty L, et al. Unpacking students' use of mathematics in upper-division physics: where do we go from here? Eur J Phys. 2015;36(6):1-28.

[8] Thompson J, Bucy B, Mountcastle D. Assessing student understanding of partial derivatives in thermodynamics. In Heron P, McCullough L and Marx J. (eds) Physics Education Research Conference, 2005.

[9] Stephan M, Rasmussen CL. Classroom mathematical practices in differential equations. J Math Behaviour. 2002;21:459-490.

[10] Redish EF, Kuo E. Language of Physics, Language of Math: Disciplinary Culture and Dynamic Epistemology. Sci Educ. 2015;24:561-590.

[11] Manogue CA, Dray T. Bridging the Gap between Mathematics and the Physical Sciences, http://www.math.oregonstate.edu/bridge/papers/bridge.pdf.

[12] Mallet DG, McCue SW. Constructive development of the solutions of linear equations in introductory ordinary differential equations. Int J Math Educ Sci 
Technol. 2009;40(5):587-595.

[13] Afasamaga-Fuata K. An undergraduate student's understanding of differential equations through concept maps and vee diagrams. In: Proceedings of the First International Conference on Concept Mapping, Pamplona, Spain, 2004.

[14] Ju MK, Kwon ON. Ways of talking and ways of positioning: students' beliefs in an inquiry-oriented differential equations class. J Math Behaviour. 2007;26:267-280.

[15] Waddy S, Kim JH, Glass M. Tutoring dialogue goals for conceptual understanding of differential equations: preliminary work. In: Proceedings of the 20th Midwest AI and Cognitive Science Conference, Indiana Purdue Fort Wayne, 2009.

[16] Raychaudhuri D. Dynamics of a definition: a framework to analyse student construction of the concept of solution to a differential equation. Int J Math Educ Sci Technol. 2009;39(2):161-177.

[17] Rasmussen CL. New directions in differential equations: A framework for interpreting students' understandings and difficulties. J Math Behaviour. 2001;20:55-87.

[18] Habre S. Investigating students' approval of a geometrical approach to differential equations and their solutions. Int J Math Educ Sci Technol. 2003;34(5):651-662.

[19] Habre S. Exploring Students' Strategies to Solve Ordinary Differential Equations in a Reformed Setting. J Math Behaviour. 2000;18(4):455-472.

[20] Rasmussen CL, Kwon ON. An inquiry-orientated approach to undergraduate mathematics. J Math Behaviour. 2007;26:189-194.

[21] Freudenthal H. Cited in Van Den Heuvel-Panhuizen M. The didactical use of models in realistic mathematics education: An example from a longitudinal trajectory on percentage. Educ Stud Math. 2003;54(1):9-35.

[22] Skemp RR. Relational Understanding and Instrumental Understanding. Math Teach. 1976;77:20-26.

[23] Tall D, Vinner S. Concept image and concept definition in mathematics with particular reference to limits and continuity. Educ Stud Math. 1981;12(2):151-169.

[24] Creswell JW, Plano Clark VL. Designing and conducting mixed methods research. 2007. Thousand Oaks.

[25] Vinner S, Dreyfus T. Images and definitions for the concept of function. J Res Math Educ. 1989;20(4):356-366. 
[26] Doughty L, McLoughlin E, van Kampen P. What integration cues, and what cues integration in intermediate electromagnetism. Am J Phys 2014;82:1093-1103.

[27] Bollen L, van Kampen P, De Cock M. Students' difficulties with vector calculus in electrodynamics. Phys Rev Physics Educ Res. 2015;11:1-14.

[28] Sfard A. On the dual nature of mathematical conceptions: Reflections on processes and objects as different sides of the same coin. Educ Stud Math. 1991;22(1):1-36.

[29] Thomas DR. A General Inductive Approach for Analyzing Qualitative Evaluation Data. Am J Eval. 2006;27(2):237-246.

[30] Bezuidenhout J. Limits and Continuity: Some Conceptions of First-Year Students. Int J Math Educ Sci Technol. 2001;32(4):487-500.

[31] University of Leeds. Faculty of engineering, 2016. [Online]. Available from: https://www.engineering.leeds.ac.uk/teaching/electronic/module/MATH1400 [Accessed 1 March 2016]

[32] McDermott LC, Shaffer PS, and the Physics Education Group at the University of Washington. Tutorials in Introductory Physics. Prentice-Hall, Upper Saddle River, NJ, 2002.

[33] McDermott LC and Shaffer PS, "Research as a guide for curriculum development: An example from introductory electricity. Part II: Design of instructional strategies,” Am J Phys. 1992;60(11):1003-1013. 